\title{
Oronasal Bleed: A Rare Case of Carotid Cavernous Sinus Fistula
}

\section{Mudit Mittal}

\section{ABSTRACT}

An intractable oronasal bleed following a craniofacial trauma due to traumatic carotid cavernous sinus fistula is an uncommon condition with very few cases reported in literature, not specific to ORL bleed. Moreover, symptoms of carotid cavernous sinus fistula usually do not develop immediately after trauma. The clinical presentations can be varied as well, mimicking diseases like multiple sclerosis, brain tumors or stroke.

The purpose of reporting this case is to emphasize the importance of early suspicion of this pathology, its clinical course and special attention to radiological studies. Here, we are reporting a successfully treated case of carotid cavernous sinus fistula with intractable oronasal bleed following a craniofacial trauma.

Keywords: Carotid cavernous sinus fistula, Nasal bleed, Trauma.

How to cite this article: Mittal M, Oronasal Bleed: A Rare Case of Carotid Cavernous Sinus Fistula. Int J Head Neck Surg 2014;5(2):87-88.

Source of support: Nil

Conflict of interest: None

\section{INTRODUCTION}

Caroticocavernous fistulas (CCFs) are abnormal connections between the carotid artery and the cavernous sinus. ${ }^{1}$ These lesions may be classified according to several criteria: angiographically, as direct (direct communication between carotid artery and cavernous sinus) or dural (dural branches of carotid artery and cavernous sinus), pathogenetically, as spontaneous or traumatic, and hemodynamically, as high flow and low flow. The dural fistulas usually have low rates of arterial blood flow and may be difficult to diagnose without angiography. ${ }^{2}$

Post-traumatic fistulas occur in approximately $0.2 \%$ of head trauma (TCEs). These lesions, high energy transmission result in changes in the course of ICA for direct communication with the cavernous sinus. This condition can result in cranial nerve palsies, blindness, subarachnoid hemorrhage, and more rarely, epistaxis, which may be massive and cause imminent life-threatening to patients. ${ }^{3}$

\section{Assistant Professor}

Department of ENT and Head and Neck Surgery, Chirayu Medical College, Bhopal, Madhya Pradesh, India

Corresponding Author: Mudit Mittal, Assistant Professor Department of ENT and Head and Neck Surgery, Chirayu Medical College, Bhopal, Madhya Pradesh, India, Phone: 09425945542 e-mail:dr_muditmittal@yahoo.co.in

\section{CASE REPORT}

A 21-year-old male with craniofacial trauma following a road traffic accident was admitted in trauma. Computed tomography (CT) scan was performed to determine intracranial lesion or skull base fracture, which was normal. The general examination of patient was normal, so as the preliminary hematology. Patient was active and was actively involved in discussions of his ailments. Two days following trauma he had massive oronasal bleeding, which stopped spontaneously after some time. At this moment no ENT call was sent as bleeding stopped spontaneously. He was discharged after another 3 days of observation. One week after discharge and symptom free period he appeared with another episode of massive oronasal bleeding and was admitted again. Blood investigations showed $\mathrm{Hb} 6.9 \mathrm{gm} \%$. Blood pressure (BP) was 70/50 $\mathrm{mm} \mathrm{Hg}$, resuscitative measures were taken and boy was saved. Nasal bleed stopped spontaneously again. Diagnostic nasal endoscopy done next day which was no help. Ophthalmologist noted a mild proptosis right but no blurry vision. Other systemic examinations were unremarkable. A contrast CT angiogram study was done which revealed significantly dilated right ophthalmic vein and ballooning of cavernous sinus suggesting carotid artery cavernous sinus fistula (Fig. 1). Patient was sent to Department of Interventational Radiology for therapeutic embolization. It is more than 5 months after the trauma patient is doing fine.

\section{DISCUSSION}

Cavernous sinuses lie on the lateral aspect of sphenoid body. These sinuses are made up of an intertwined network of thin walled venous channels of varying sizes that divide and coalesce incompletely, surround the carotid arteries. ${ }^{4}$
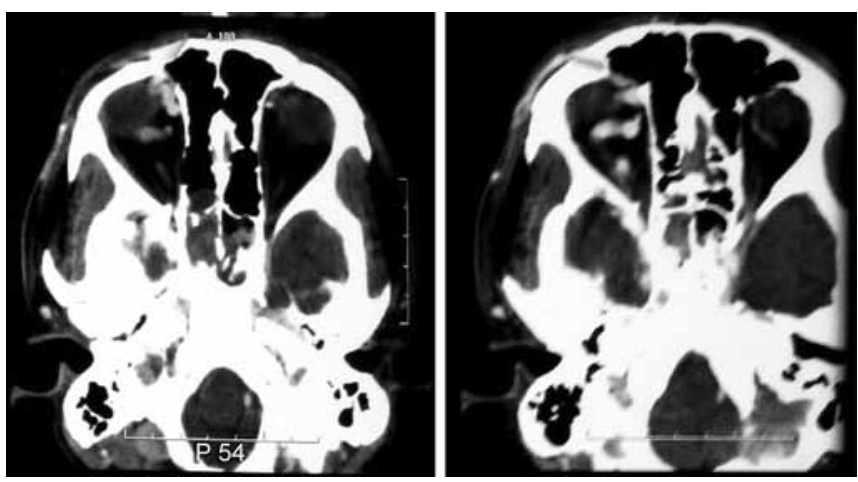

Fig. 1: Carotid cavernous fistulas 
Caroticocavernous fistulas can be categorized using the Barrow classification system. Type A fistulas involve a direct communication between the intercavernous portion of the internal carotid artery (ICA) and the cavernous sinus, whereas types $\mathrm{B}, \mathrm{C}$ and $\mathrm{D}$ are indirect communications between either dural branches of the ICA or external carotid artery (ECA) and the cavernous sinus. Barrows type A fistula is most common in cases of trauma. But Dural CCF (Barrows B-D) may occur. ${ }^{1}$

Approximately $75 \%$ of all reported FCCS are caused by trauma due to severe injuries, such as car accidents or penetrating trauma. ${ }^{2}$ The case we are reporting here has a similar history of blunt trauma. Despite this, there are reports of minimal facial trauma that can develop CCF. ${ }^{5}$

Symptom onset is typically delayed until venous hypertension reaches a critical level, often days to weeks following the initial insult. In this case also the episode of first epistaxis was after 15 days of head trauma, later other symptoms like proptosis of right eye with mild headache gradually developed, although no cranial nerve palsy observed, this can be attributed to the fact as postulated, the CCF occurs when there is disruption of intracavernous ICA resulting in abnormal communication. The voluminous flow that passes through the fistula overloads and dilates the cavernous sinus, causing hypertension and reversal of flow to the ophthalmic veins, leads to exophthalmos, cranial nerve palsies, blindness and orbital pain. Venous hypertension in dural sinuses can lead to in rare case the appearance of epistaxis - sometimes massive and occasionally fatal. The increased venous pressure and blood within the cavernous sinus and its expansive nature cause erosion of the thin walls of the sphenoid sinus, leading to extravasation of intravascular contents into the sphenoid sinus cavity. ${ }^{6}$

Angiography is the test of choice for definitive diagnosis. Angiographic demonstration of a traumatic CCF, either the direct variety or the dural type, from the day of trauma is rare, because symptoms of traumatic CCF usually do not appear immediately after trauma and angiography is rarely used in the diagnosis of acute head trauma. ${ }^{7}$ In this case, a contrast $\mathrm{CT}$ angiogram was done which showed significantly dilated right ophthalmic vein which can be due to the retrograde flow in the ophthalmic vein due to the change in flow of direction of blood within the cavernous sinus. The conven- tional treatment for transarterial CCF is embolization ICA by introducing a microcatheter. This method is successful in 85 to $97 \%$ of cases. Complications occur in 1 to $3 \%$ of cases and are usually attributed to the migration of balloons or embolism resulting in stroke. ${ }^{6}$ In this case also selective embolization was done by interventional radiologist and no episode of further nasal bleed was recorded for another 15 days, thus patient was discharged from hospital. Till date the patient is symptom free.

In case of failure of this procedure, surgically complete occlusion of the left ICA, by metal clips is other option.

\section{CONCLUSION}

The CCF is unusual complication after craniofacial trauma, and the concomitant presence of massive epistaxis makes the case unusual should alert the otolaryngologist to the possibility of a uncontrollable bleeding.

Reorganization of traumatic CCF is important as it allows proper selection of treatment strategies. Although, their spontaneous disappearance is known, some of these do become symptomatic and need treatment as these can progress to intracranial hemorrhage, vision loss, and even life-threatening epistaxis.

\section{REFERENCES}

1. Barrow DL, Spector RH, Braun IF. Classification and treatment of spontaneous carotid-cavernous sinus fistulas. J Neurosurg 1985 Feb;62(2):248-256.

2. Chaudhry IA, Elkhamry SM, Al-Rashed W, Bosley TM. Carotid cavernous fistula: ophthalmological implications. Middle East Afr J Ophthalmol 2009 Apr;16(2):57-63.

3. Millman B, Giddings NA. Traumatic carotid-cavernous sinus fistula with delayed epistaxis. Ear Nose Throat J 1994 June;73(6): 408-411.

4. Hill J. The pituitary gland, anatomy and physiology. Scott Brown's Otolaryngology and Head Neck Surgery. In: Gleeson M, editor. London: Edward Arnold Ltd, 2008;7th ed, volume 3. p. 299.

5. Keiser GJ, Zeidman A, Gold BD. Carotid-cavernous fistula after minimal facial trauma. Oral Surg Oral Med Oral Pathol 1991; 71(5):549-551.

6. Borba R, Sonda I, Dini LI, Calcagnotto FN, Marchett N, Cobalchini PC. Carotid-cavernous fistula with lethal epistaxis: case report. Arq Neuropsiquiatr 2001;59(2-A):276-279.

7. Komiyama M, Nakajima H, Nishikawa M, Kan M. Traumatic carotid cavernous sinus fistula: serial angiographic studies from the day of trauma. AJNR 1998 Oct;19:1641-1644. 